\title{
OPEN Impact of exercise capacity on the long-term incidence of atrial arrhythmias in heart failure
}

\author{
Tetsuri Sakai ${ }^{1,2}$, Atsuhiko Yagishita ${ }^{1,2 \bowtie}$, Masahiro Morise ${ }^{1}$, Susumu Sakama ${ }^{1}$, Takeshi ljichi $^{1}$, \\ Kengo Ayabe ${ }^{1}$, Mari Amino ${ }^{1}$, Yuji lkari ${ }^{1}$ \& Koichiro Yoshioka ${ }^{1}$
}

We sought to demonstrate the impact of improved peak exercise oxygen consumption $\left(\mathrm{VO}_{2}\right)$ during maximal exercise testing after cardiac rehabilitation (CR) on the incidence of arrhythmias in patients with heart failure (HF). The present study comprised of 220 patients with $\mathrm{HF}$, and peak $\mathrm{VO}_{2}$ was examined at 2 and 5 months after CR. Of the 220 patients, $110(50 \%)$ had a low peak $\mathrm{VO}_{2}$ of $<14 \mathrm{~mL}$ / $\mathrm{min} / \mathrm{kg}$ at 2 months. The peak $\mathrm{VO}_{2}$ improved in 86 of these $110(78 \%)$ patients at 5 months after CR. During a median follow-up of 6 years, the patients with improvement in peak $\mathrm{VO}_{2}$, compared to those without peak $\mathrm{VO}_{2}$ improvement, had a lower rate of mortality ( $4 \% \mathrm{vs.} 29 \%$, log-rank, $\left.P<0.001\right)$ and $\mathrm{HF}$ hospitalization ( 6 vs. $17 \%$, log-rank, $P=0.044)$ and a lower incidence of new-onset atrial arrhythmias ( 9 vs. $27 \%$, log-rank, $P=0.013$ ), with no difference in the incidence of ventricular arrhythmias between groups (1 vs. $4 \%$, log-rank, $P=0.309$ ). The majority of deaths in the patients without an improved peak $\mathrm{VO}_{2}$ were because of cardiovascular events $(73 \%)$, particularly progressive HF (55\%). Early detection and management of atrial arrhythmias may improve outcomes in patients without peak $\mathrm{VO}_{2}$ improvement after CR.

Reduced exercise capacity is a principal symptom of heart failure (HF). Determination of peak exercise oxygen consumption $\left(\mathrm{VO}_{2}\right)$ during maximal exercise testing has been used to assess exercise capacity in patients with HF. Mancini et al. demonstrated that a low peak $\mathrm{VO}_{2}$ of $<14 \mathrm{~mL} / \mathrm{kg} / \mathrm{min}$ was associated with poor prognosis in patients with severe HF (higher-risk) ${ }^{1}$. Improvement in exercise capacity is one of the most important effects of exercise training in cardiac rehabilitation $(\mathrm{CR})^{2-5}$, leading to a better outcome in patients with ischemic $\mathrm{HF}^{6-9}$. Although atrial and ventricular arrhythmias contribute to the morbidity and mortality of patients with $\mathrm{HF}^{3}$, the impact of improvement in exercise capacity after CR on the long-term incidence of atrial and ventricular arrhythmias remains uncertain. We hypothesized that improvement in peak $\mathrm{VO}_{2}$ after $\mathrm{CR}$ would reduce the risk of long-term incidence of cardiac arrhythmias and mortality in patients with HF. In the present study, we sought to determine the association between the changes in exercise capacity after CR and the incidence of new-onset atrial and ventricular arrhythmias and the rate of mortality and hospitalization due to HF exacerbation during long-term follow-up.

\section{Methods}

Study design and participants. In this retrospective single-center study, 220 consecutive patients with HF who underwent CR at the Tokai University Hospital (Kanagawa, Japan) from April 2012 to March 2016 were included in the study. Patients with acute HF or exacerbation of chronic HF participated in the CR programs during the acute phase once the patients' condition was stabilized with pharmacological and/or non-pharmacological interventions ${ }^{4}$. Patients who were not eligible for CR included those with acute coronary syndrome before non-pharmacological interventions, refractory arrhythmias, uncontrolled HF (e.g., New York Heart Association [NYHA] Class IV), and symptomatic severe aortic stenosis before surgical interventions, as well as those unable to provide consent to CR. Patients were classified into two groups: those with a low peak $\mathrm{VO}_{2}$ (higher-risk patients) and those without a low peak $\mathrm{VO}_{2}$ (lower-risk patients) at 2 months after the start of CR. Furthermore, higher-risk patients were subdivided into two groups: higher-risk patients with an improved peak $\mathrm{VO}_{2}$ at 5 months and those without improvement in the peak $\mathrm{VO}_{2}$. Improvement in the exercise capacity was defined as the increase in the peak $\mathrm{VO}_{2}$ after the CR. The clinical characteristics, the incidence of new-onset atrial arrhythmias including atrial fibrillation (AF) and atrial tachycardia, and ventricular arrhythmias including ventricular tachycardia and ventricular fibrillation, and the rate of mortality and hospitalization due to HF 
exacerbation were determined among higher-risk patients. The present study was approved by the Institutional Review Board for Clinical Research, Tokai University (20R-058). The board waived the condition of obtaining informed consent for study participation. All methods included in this study were carried out in accordance with relevant guidelines and regulations.

Cardiopulmonary exercise testing. Patients underwent symptom-limited cardiopulmonary exercise testing (CPX) using a cycle ergometer (Lode, Groningen, Netherlands) with respiratory gas exchange analysis, when they were clinically stable, at 2 and 5 months during the CR program. The CPX protocol consisted of the following: 4-min rest period while sitting on the cycle ergometer; 2 -min exercise at the free-wheel; and an incremental exercise phase with an increase of $10 \mathrm{~W} / \mathrm{min}$ (ramp protocol). A 12-lead electrocardiogram was continuously monitored throughout the test (Nihon Kohden Corporation, Tokyo, Japan). Breath-by-breath expiratory gas analysis was measured, and measured minute ventilation, oxygen uptake $\left(\mathrm{VO}_{2}\right)$, and carbon dioxide production data were stored on a computer hard disk every $6 \mathrm{~s}$ for off-line analysis (Minato Medical Science, Osaka, Japan). Peak $\mathrm{VO}_{2}$ was defined as the highest $\mathrm{VO}_{2}$ during exercise testing, which was expressed as a value adjusted to body weight $(\mathrm{mL} / \mathrm{kg} / \mathrm{min})$. A low peak $\mathrm{VO}_{2}$ was defined as $<14 \mathrm{~mL} / \mathrm{min} / \mathrm{kg}^{1}$. Predicted values of $\mathrm{VO}_{2}$ was calculated as follows: peak VO2 pred $=(\text { Height }- \text { Age })^{\star} 20$ if male, $=(\text { Height }- \text { Age })^{\star} 14$ if female ${ }^{10}$.

Statistical analysis. Categorical variables are expressed as absolute and relative frequencies. For categorical data, Fisher's exact test was applied. Quantitative variables were described by their mean ( \pm standard deviation [SD]) or by the median and interquartile range (IQR), as appropriate for their distribution, and compared using the Mann-Whitney U test. Survival curves were estimated using the Kaplan-Meier method and compared using log-rank tests. We performed univariate Cox regression analysis of clinical outcomes in association with improvement in peak $\mathrm{VO}_{2}$ with $\mathrm{CR}$ in higher-risk patients. A 2-tailed $p$ value of $<0.05$ was considered to indicate statistical significance. All statistical analyses were performed using SPSS Statistics version 23.0 (IBM Corp, Armonk, NY, USA).

\section{Results}

Patient characteristics. The median age of the study group was 67 (IQR, 59-74) years, including 51 women (23\%); the median plasma brain natriuretic peptide level was 215.2 (IQR, 104.1-494.7) pg/ml. The majority of patients had an NYHA class I, with optimal medical therapy including angiotensin-converting enzyme inhibitors or angiotensin II receptor blockers and beta-blockers, at the 2-month time point of CR.

Of the 220 patients included, 110 had a low peak $\mathrm{VO}_{2}(50 \%)$ at 2 months of CR (higher-risk patients, Table 1). Compared to lower-risk patients, the proportion of female patients was higher $(30 \% \mathrm{vs.} 16 \%, P=0.025)$ and the median left ventricular ejection fraction was lower ( $49 \%$ vs. $54 \%$, respectively, $P=0.019$ ). In 117 patients with preserved ejection fraction on echocardiography, the cause of heart failure was not different between the two groups: ischemic in $69 \%$ in higher-risk patients vs. $83 \%$ in lower-risk patients $(P=0.086)$. The mean values of peak $\mathrm{VO}_{2}$ at 2 months were $11.8 \pm 1.8 \mathrm{~mL} / \mathrm{min} / \mathrm{kg}$ in higher-risk patients and $17.4 \pm 2.7 \mathrm{~mL} / \mathrm{min} / \mathrm{kg}$ in lower-risk patients, respectively $(P<0.001)$. The mean values of peak $\mathrm{VO}_{2}$ at 5 months were $14.2 \pm 3.7 \mathrm{~mL} / \mathrm{min} / \mathrm{kg}$ in higherrisk patients and $19.0 \pm 4.5 \mathrm{~mL} / \mathrm{min} / \mathrm{kg}$ in lower-risk patients, respectively $(P<0.001)$. Peak $\mathrm{VO}_{2}$ percentage of predicted value was $43.2 \pm 9.8 \%$ in higher-risk patients and $57.5 \pm 10.7 \%$ in the lower-risk patients, respectively $(P<0.001)$.

Among the 110 higher-risk patients, the peak $\mathrm{VO}_{2}$ improved in $86(78 \%)$ at 5 months of CR. There were no differences in clinical characteristics between higher-risk patients with improvement in peak $\mathrm{VO}_{2}$ and those without (Table 2), except for the peak $\mathrm{VO}_{2}$ at 5 months $(10.8 \pm 1.8$ vs. $15.1 \pm 3.5 \mathrm{~mL} / \mathrm{min} / \mathrm{kg}, P<0.001)$.

Long-term outcomes. During a median follow-up of 6 years, higher-risk patients with improvement in peak $\mathrm{VO}_{2}$, compared to those without peak $\mathrm{VO}_{2}$ improvement, had a lower rate of mortality (4\% vs. 29\%; logrank, $P<0.001$, Fig. 1a) and hospitalization due to HF exacerbation (6\% vs. $17 \%$; log-rank, $P=0.044$, Fig. 1b). Unadjusted hazard ratios of mortality and hospitalization for higher-risk patients with an improved peak $\mathrm{VO}_{2}$ with CR were 0.132 (95\% confidence interval [CI] 0.034-0.511; $P=0.003)$ and 0.156 (95\% CI 0.018-0.1373; $P=0.094)$, respectively. Among 196 patients without prior AF, 23 patients (12\%) had atrial arrhythmias: AF in 18 patients and atrial tachycardia in five. Higher-risk patients with improvement in peak $\mathrm{VO}_{2}$ with $\mathrm{CR}$ had a lower incidence of new-onset atrial arrhythmias than did those without a peak $\mathrm{VO}_{2}$ improvement (9\% vs. $27 \%$; logrank, $P=0.013$, Fig. 2a). Unadjusted hazard ratio of new-onset atrial arrhythmias for patients with an improved peak VO with CR was $0.263(95 \%$ CI $0.085-0.830 ; P=0.021)$. New-onset ventricular arrhythmias occurred in four patients: ventricular tachycardia in three patients and ventricular fibrillation in one. There was no difference in the incidence of ventricular arrhythmias between higher-risk patients with and without improvement in peak $\mathrm{VO}_{2}$ with $\mathrm{CR}(1 \%$ vs. $4 \%$; log-rank, $P=0.309$, Fig. $2 \mathrm{~b})$. Unadjusted hazard ratio of new-onset ventricular arrhythmias for higher-risk patients with an improved peak $\mathrm{VO}_{2}$ with CR was 0.262 (95\% CI 0.016-4.195; $P=0.344$ ). Patients with peak $\mathrm{VO}_{2}$ percentage of predicted values of $<45 \%$ had a higher risk of mortality (logrank, $P=0.010$ ) and higher incidence of new-onset atrial arrhythmias than did those with peak $\mathrm{VO}_{2}$ percentage of predicted values of $\geq 45 \%$ (log-rank, $P=0.031$ ), though no differences were found in hospitalization due to $\mathrm{HF}$ exacerbation (log-rank, $P=0.081)$ and new-onset ventricular arrhythmias (log-rank, $P=0.229$ ).

Table 3 summarizes the causes of death among the 11 patients with a low peak $\mathrm{VO}_{2}$. Eight of these 11 patients died from cardiovascular events (73\%), with progressive HF being the predominant cause (6 of 11 patients, $55 \%)$. In these cases, there was no incidence of thromboembolic events. Of the eight patients who died from cardiovascular events, three had new-onset atrial arrhythmias (38\%). None of the higher-risk patients underwent catheter ablation for new-onset atrial arrhythmias. 


\begin{tabular}{|c|c|c|c|c|}
\hline & All $(n=220)$ & Lower-risk patients $(\mathrm{n}=110)$ & Higher-risk patients $(\mathrm{n}=110)$ & $P$ value \\
\hline Median age (IQR), years & $67(59-74)$ & $65(58-71)$ & $68(62-76)$ & 0.110 \\
\hline Female sex, N (\%) & $51(23)$ & $18(16)$ & $33(30)$ & 0.025 \\
\hline Median body mass index (IQR), $\mathrm{kg} / \mathrm{m}^{2}$ & $23.5(20.8-25.2)$ & $23.4(20.5-25.2)$ & $23.5(21.0-25.4)$ & 0.625 \\
\hline Median BNP level (IQR), pg/ml & $215.2(104.1-494.7)$ & $213.9(103.6-364.8)$ & $217.6(105.6-618.7)$ & 0.294 \\
\hline $\begin{array}{l}\text { Median left ventricular ejection frac- } \\
\text { tion (IQR), \% }\end{array}$ & $50(41-60)$ & $54(43-61)$ & $49(40-56)$ & 0.019 \\
\hline $\begin{array}{l}\text { Median estimated GFR(IQR), ml/ } \\
\mathrm{min} / 1.73 \mathrm{~m}^{2}\end{array}$ & $63.0(52.0-77.8)$ & $65.0(56.0-75.5)$ & $61.0(46.0-78.3)$ & 0.141 \\
\hline Peak VO2 at 2 months & $14.6 \pm 3.6$ & $17.4 \pm 2.7$ & $11.8 \pm 1.8$ & $<0.001$ \\
\hline Peak VO2 at 5 months & $16.6 \pm 4.7$ & $19.0 \pm 4.5$ & $14.2 \pm 3.7$ & $<0.001$ \\
\hline Peak $\mathrm{VO}_{2}$ percentage of predicted value & $50.3 \pm 12.5$ & $57.5 \pm 10.7$ & $43.2 \pm 9.8$ & $<0.001$ \\
\hline NYHA class -N. (\%) & & & & 0.066 \\
\hline I & $147(67)$ & $80(73)$ & $67(61)$ & \\
\hline II & $57(26)$ & $24(22)$ & $33(30)$ & \\
\hline III & $16(7)$ & $6(6)$ & $10(9)$ & \\
\hline \multicolumn{5}{|l|}{ Coexisting conditions, N (\%) } \\
\hline Hypertension & $152(69)$ & $81(74)$ & $71(65)$ & 0.189 \\
\hline Diabetes & $77(35)$ & $39(36)$ & $38(35)$ & 1.000 \\
\hline Atrial fibrillation & $24(11)$ & $7(6)$ & $17(16)$ & 0.050 \\
\hline Prior thromboembolic events & $5(2)$ & $2(2)$ & $3(3)$ & 1.000 \\
\hline CHADS2 score (IQR) & $2(2-3)$ & $2(2-3)$ & $2(2-3)$ & 0.756 \\
\hline CHA2DS2-VASc (IQR) & $4(3-4)$ & $4(3-4)$ & $4(3-5)$ & 0.942 \\
\hline $\begin{array}{l}\text { Heart failure with preserved ejection } \\
\text { fraction, } \mathrm{N}(\%)\end{array}$ & $117(53)$ & $63(57)$ & $54(49)$ & 0.280 \\
\hline Cause of heart failure, $\mathrm{N}(\%)$ & & & & 0.878 \\
\hline Ischemic & $159(72)$ & $79(72)$ & $80(73)$ & \\
\hline \multicolumn{5}{|l|}{ Non-ischemic } \\
\hline Idiopathic & $31(14)$ & $17(16)$ & $14(13)$ & \\
\hline Valvular & $30(14)$ & $14(6)$ & $16(7)$ & \\
\hline \multicolumn{5}{|l|}{ Medications, N (\%) } \\
\hline ACE inhibitor or ARB & $200(91)$ & $100(91)$ & $100(91)$ & 1.000 \\
\hline Beta-blocker & $177(81)$ & $90(82)$ & $87(79)$ & 0.734 \\
\hline Amiodarone & $11(5)$ & $5(5)$ & $6(6)$ & 1.000 \\
\hline Preexisting pacemaker or CRT, N (\%) & $8(4)$ & $5(5)$ & $3(3)$ & 0.486 \\
\hline
\end{tabular}

Table 1. Clinical characteristics of the enrolled patients. IQR; interquartile range, GFR; glomerular filtration rate, NYHA; New York Heart Association, ACE; angiotensin-converting enzyme, ARB; angiotensin II receptor blocker, CRT; cardiac resynchronization therapy, BNP; brain natriuretic peptide, GFR; glomerular filtration rate.

\section{Discussion}

Our major findings were as follows: (1) the majority of patients with a low peak $\mathrm{VO}_{2}$ (higher-risk patients), defined as $<14 \mathrm{~mL} / \mathrm{min} / \mathrm{kg}$, had improvement in peak $\mathrm{VO}_{2}$ at 3 months after CR; (2) higher-risk patients with an improved peak $\mathrm{VO}_{2}$ had a lower rate of mortality and hospitalization due to HF exacerbation, as well as a lower incidence of new-onset atrial arrhythmias than did those without improvement in peak $\mathrm{VO}_{2}$, but with no difference in the incidence of ventricular arrhythmias between groups; (3) patients with peak $\mathrm{VO}_{2}$ percentage of predicted values of $<45 \%$ had a higher risk of mortality (log-rank, $P=0.010)$ and higher incidence of new-onset atrial arrhythmias than did those with peak $\mathrm{VO}_{2}$ percentage of predicted values of $\geq 45 \%$ (log-rank, $P=0.031$ ); and (4) the majority of deaths in higher-risk patients were cardiovascular events, with progressive HF being predominant.

Impaired exercise capacity is a major symptom in patients with $\mathrm{HF}$, which is represented by low peak $\mathrm{VO}_{2}$ during maximal exercise testing. A previous study by Mancini et al. demonstrated that a low peak $\mathrm{VO}_{2}$ of $<14 \mathrm{~mL} /$ $\mathrm{kg} / \mathrm{min}$ was associated with poor prognosis in patients with severe $\mathrm{HF}^{1}$. A comprehensive CR program includes patient evaluation, exercise training, physical activity counseling, cardiovascular risk factor management, psychosocial support, and patient education ${ }^{3,5}$. Previous studies demonstrated that CR improved peak $\mathrm{VO}_{2}$ and decreased all-cause mortality in patients with $\mathrm{HF}^{5,9,11-13}$. The HF-ACTION trial demonstrated the positive impact of CR on the long-term cardiovascular mortality or HF hospitalization during a median follow-up of 30 months $^{13}$, which was in accordance with our findings. However, long-term outcomes of higher-risk patients without improvement in peak $\mathrm{VO}_{2}$ despite a comprehensive CR program have not been fully investigated, particularly regarding the long-term incidence of atrial and ventricular arrhythmias in those patients. In this study, higher-risk patients without improvement in peak $\mathrm{VO}_{2}$ after $\mathrm{CR}$ had a higher rate of mortality, HF hospitalization, 


\begin{tabular}{|c|c|c|c|}
\hline & $\begin{array}{l}\text { Patients with improvement in peak } \\
\text { VO2 }(n=86)\end{array}$ & $\begin{array}{l}\text { Patients without improvement in peak } \\
\text { VO2 }(n=24)\end{array}$ & $P$ value \\
\hline Median age (IQR), years & $67(60-76)$ & $70(65-78)$ & 0.192 \\
\hline Female sex, $\mathrm{N}(\%)$ & $28(33)$ & $5(21)$ & 0.322 \\
\hline Median body-mass index (IQR), $\mathrm{kg} / \mathrm{m}^{2}$ & $23.1(20.9-25.1)$ & $24.4(21.5-27.2)$ & 0.178 \\
\hline Median BNP level (IQR), pg/ml & $208.7(104.0-513.1)$ & $437.5(124.9-751.2)$ & 0.147 \\
\hline $\begin{array}{l}\text { Median left ventricular ejection fraction } \\
\text { (IQR), \% }\end{array}$ & $50(41-57)$ & $44(34-53)$ & 0.172 \\
\hline $\begin{array}{l}\text { Median estimated GFR (IQR), } \mathrm{ml} / \\
\mathrm{min} / 1.73 \mathrm{~m}^{2}\end{array}$ & $62.5(48.8-79.0)$ & $51.5(37.5-71.8)$ & 0.074 \\
\hline Peak VO2 at 2 months & $11.7 \pm 1.9$ & $12.0 \pm 1.4$ & 0.682 \\
\hline Peak VO2 at 5 months & $15.1 \pm 3.5$ & $10.8 \pm 1.8$ & $<0.001$ \\
\hline NYHA class -N. (\%) & & & 0.457 \\
\hline I & $55(64)$ & $12(50)$ & \\
\hline II & $24(28)$ & $9(38)$ & \\
\hline III & $7(8)$ & $3(13)$ & \\
\hline \multicolumn{4}{|l|}{ Coexisting conditions, N (\%) } \\
\hline Hypertension & $58(67)$ & $13(54)$ & 0.239 \\
\hline Diabetes & $30(35)$ & $8(33)$ & 1.000 \\
\hline Atrial fibrillation & $15(17)$ & $2(8)$ & 0.354 \\
\hline Prior thromboembolic events & $2(2)$ & $1(4)$ & 0.526 \\
\hline CHADS2 score (IQR) & $2(2-3)$ & $2(2-3)$ & 0.391 \\
\hline CHA2DS2-VASc (IQR) & $4(3-5)$ & $4(3-5)$ & 0.673 \\
\hline $\begin{array}{l}\text { Heart failure with preserved ejection } \\
\text { fraction, } \mathrm{N}(\%)\end{array}$ & $45(52)$ & $8(33)$ & 0.112 \\
\hline Cause of heart failure, $\mathrm{N}(\%)$ & & & 1.000 \\
\hline Ischemic & $63(73)$ & $17(71)$ & \\
\hline Non-ischemic & $8(9)$ & $6(25)$ & \\
\hline Idiopathic & $15(17)$ & $1(4)$ & \\
\hline \multicolumn{4}{|l|}{ Valvular } \\
\hline \multicolumn{4}{|l|}{ Medications, N (\%) } \\
\hline ACE inhibitor or ARB & $77(90)$ & $23(96)$ & 0.688 \\
\hline Beta-blocker & $71(83)$ & $16(67)$ & 0.153 \\
\hline Amiodarone & $5(6)$ & $1(4)$ & 1.000 \\
\hline Preexisting pacemaker or CRT, N (\%) & $3(4)$ & 0 & 1.000 \\
\hline
\end{tabular}

Table 2. Clinical characteristics of the higher-risk patients. IQR; interquartile range, GFR; glomerular filtration rate, NYHA; New York Heart Association, ACE; angiotensin-converting enzyme, ARB; angiotensin II receptor blocker, CRT; cardiac resynchronization therapy, BNP; brain natriuretic peptide.

and new-onset atrial arrhythmias than those with improvement. Of note, the majority of deaths were progressive HF, suggesting that most patients with severe HF, who are more prone to develop atrial arrhythmias, easily evolve towards advanced $\mathrm{HF}$.

HF predisposes to the occurrence of atrial arrhythmias through various mechanisms, including an increase in left ventricular filling pressure or left atrial dilatation and fibrosis, each of which can lead to atrial structural and electrical remodeling ${ }^{14}$. Conversely, atrial arrhythmias, with an increased heart rate, can predispose to the development or worsening of HF due to impaired contractility and reduced cardiac output. Therefore, the occurrence of atrial arrhythmias in patients with HF is associated with increased adverse events, including HF progression and mortality ${ }^{15,16}$. In our study, the majority of deaths of higher-risk patients without improvement in peak $\mathrm{VO}_{2}$, who were at high risk for new-onset atrial arrhythmias, were cardiovascular events, mostly progressive HF, which was in accordance with previous studies. Although the treatment of atrial arrhythmias in patients with HF is of pivotal importance, previous randomized trials have failed to demonstrate that maintenance of sinus rhythm with antiarrhythmic drug therapy improves mortality in patients with coexisting $\mathrm{HF}$ and $\mathrm{AF}^{17}$, suggesting that side effects of antiarrhythmic drugs offset the positive effects. Recent studies have shown that catheter ablation is associated with a positive outcome in comparison with antiarrhythmic drugs ${ }^{18-20}$. The CASTLE-AF (Catheter Ablation versus Standard Conventional Therapy in Patients with Left Ventricular Dysfunction and Atrial Fibrillation) trial showed that catheter ablation was associated with lower rates of death from any cause and lower rates of hospital admission for HF in patients with HF. In a sub-analysis of the CAMERA MRA study, a regression of ventricular fibrosis in the context of reverse remodeling was observed on cardiac magnetic resonance imaging after restoration of sinus rhythm following catheter ablation of $\mathrm{AF}$ in patients with $\mathrm{HF}^{21}$, suggesting that timely treatment with catheter ablation may minimize irreversible ventricular remodeling. Although none of 

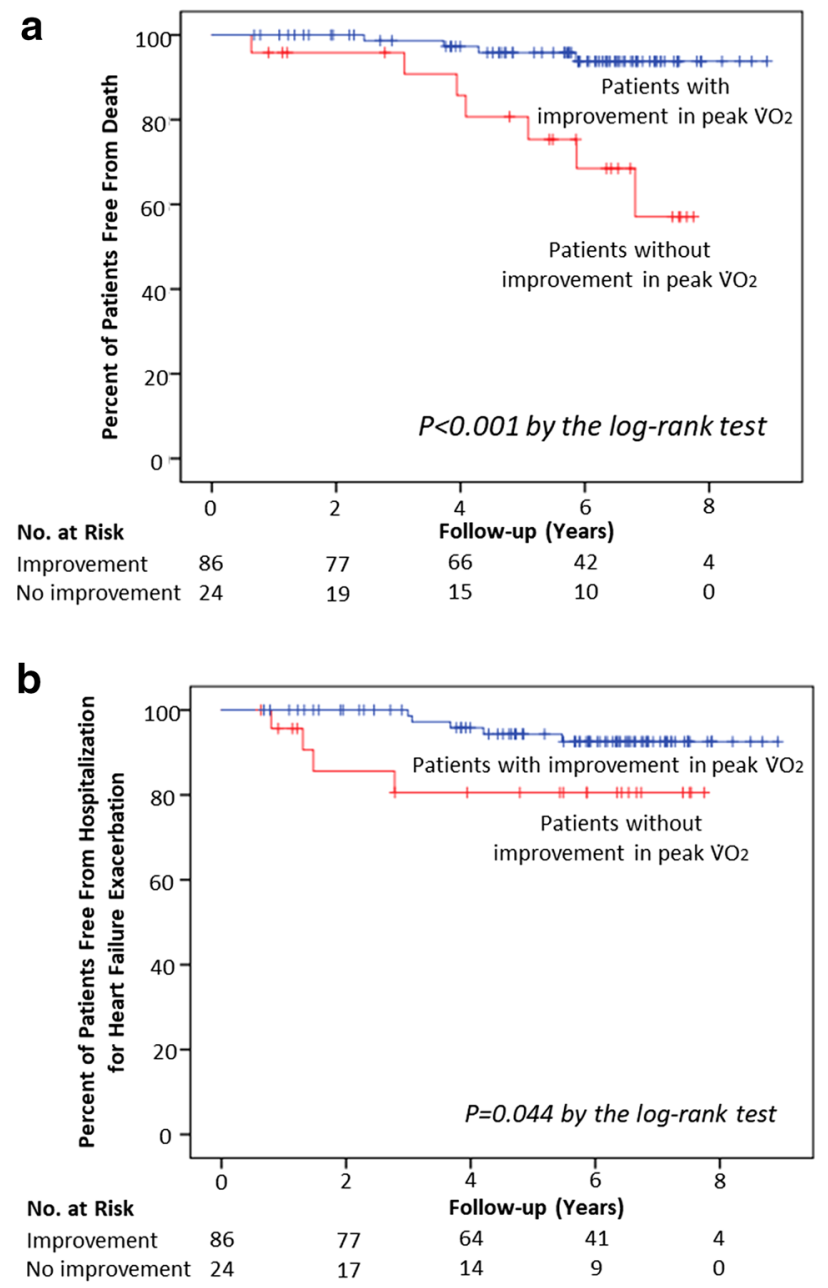

Figure 1. Mortality (a) and hospitalization due to heart failure exacerbation (b) over time. The Kaplan-Meier estimates of mortality and hospitalization for HF exacerbation over a median follow-up of 6 years are shown for high-risk patients with improvement in peak $\mathrm{VO}_{2}$, high-risk patients without improvement in peak $\mathrm{VO}_{2}$, and patients with a preserved $\mathrm{VO}_{2}$. Tick marks indicate censored data. $\mathrm{HF}$, heart failure; $\mathrm{VO}_{2}$, peak exercise oxygen consumption.

the deceased higher-risk patients with new-onset atrial arrhythmias underwent catheter ablation in this study, catheter ablation at the early stage of new-onset atrial arrhythmias may have improved the long-term outcome ${ }^{22}$.

\section{Limitations}

First, this was a retrospective single-center study with a limited number of patients since we aimed to include those who were able to be followed for a long-term period, which was possibly associated with the low incidence of new-onset ventricular arrhythmias. Furthermore, due to the limited number of patients, multivariate analysis was not performed in this study. Further multicenter investigation in a large number of patients is warranted. Second, as in many other countries, the number and duration of supervised CR sessions were limited due to a reimbursement policy in Japan. The prolonged CR may have further improved peak $\mathrm{VO}_{2}$, as observed in a previous study ${ }^{23}$, particularly in higher-risk patients. Third, considering that most patients enrolled in this study were on NYHA class I, with optimal therapies at the 2-month time point of CR, our data can be applied only to patients without severe HF who are refractory to medical and interventional therapies. Fourth, due to the lack of a predefined protocol in this retrospective study, systematic follow-up echocardiographic evaluation was not performed in this study to assess the relationship between the peak $\mathrm{VO}_{2}$ improvement and changes in echocardiographic parameters.

\section{Conclusion}

Improvement in peak $\mathrm{VO}_{2}$ after $\mathrm{CR}$ reduced the risk of new-onset atrial arrhythmias, mortality, and hospitalization due to HF exacerbation in higher-risk patients with HF. Further studies are warranted to determine whether early detection and management at the early stage of new-onset atrial arrhythmias could improve clinical outcomes in higher-risk patients without improvement in exercise capacity after CR. 

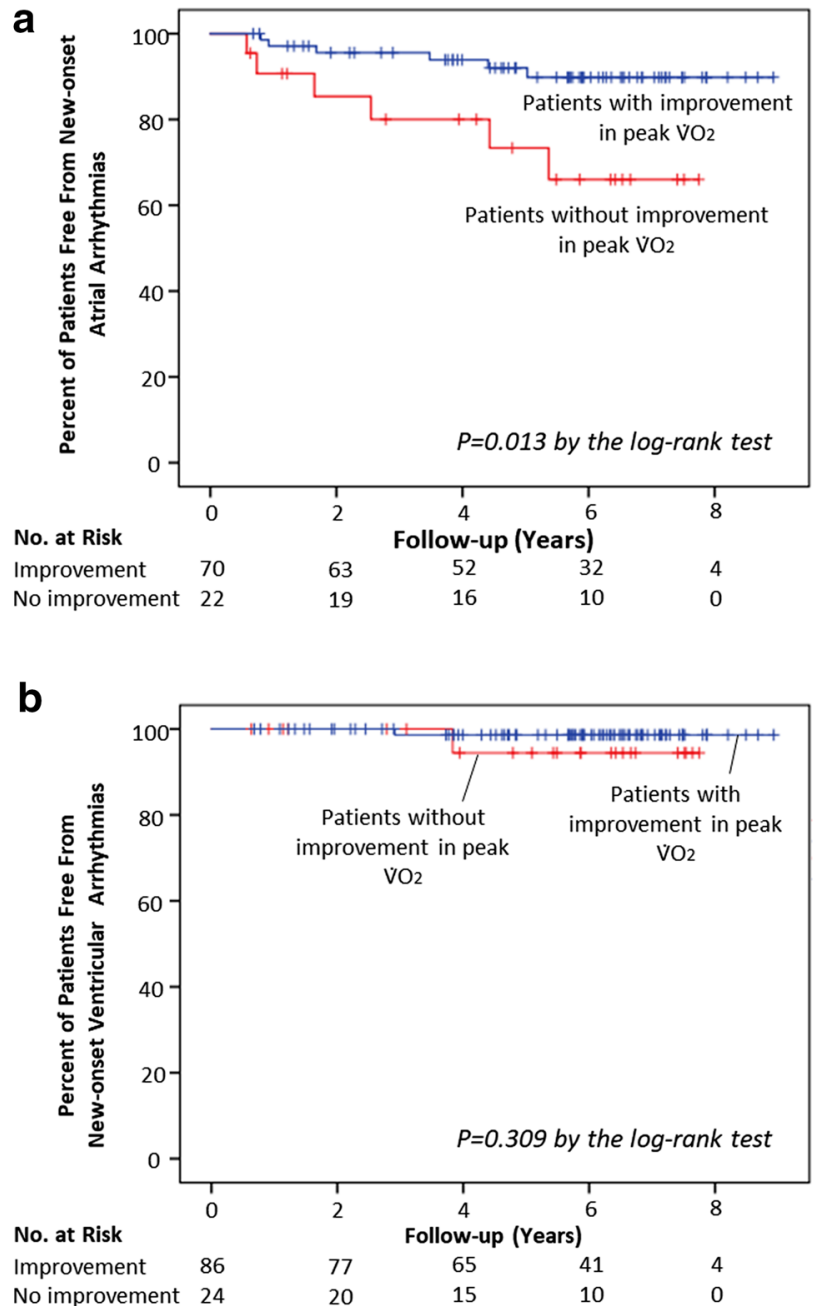

Figure 2. New-onset atrial (a) and ventricular (b) arrhythmias over time. The Kaplan-Meier estimates of the incidence of new-onset atrial and ventricular arrhythmias over a median follow-up of 6 years are shown for high-risk patients with improvement in peak $\mathrm{VO}_{2}$, high-risk patients without improvement in peak $\mathrm{VO}_{2}$, and patients with a preserved $\mathrm{VO}_{2}$. Tick marks indicate censored data. $\mathrm{HF}$, heart failure; $\mathrm{VO}_{2}$, peak exercise oxygen consumption.

\begin{tabular}{|l|l|}
\hline \multicolumn{2}{|l|}{ No. (\%) } \\
\hline Total & 11 \\
\hline Cardiovascular death & $8(73)$ \\
\hline Cardiac & $2(18)$ \\
\hline Sudden cardiac death & $6(55)$ \\
\hline Progressive heart failure & Vascular \\
\hline Thromboembolic events & 0 \\
\hline Hemorrhage & 0 \\
\hline Non-cardiovascular death & $3(27)$ \\
\hline Cancer & $1(9)$ \\
\hline Infection & $2(18)$ \\
\hline
\end{tabular}

Table 3. Causes of death in the higher-risk patients without improvement in peak VO2. 


\section{Data availability}

The datasets generated during and/or analyzed during the current study are available from the corresponding author on reasonable request.

Received: 14 June 2021; Accepted: 6 September 2021

Published online: 21 September 2021

\section{References}

1. Mancini, D. M. et al. Value of peak exercise oxygen consumption for optimal timing of cardiac transplantation in ambulatory patients with heart failure. Circulation 83, 778-786 (1991).

2. van Tol, B. A., Huijsmans, R. J., Kroon, D. W., Schothorst, M. \& Kwakkel, G. Effects of exercise training on cardiac performance, exercise capacity and quality of life in patients with heart failure: A meta-analysis. Eur. J. Heart Fail. 8, 841-850 (2006).

3. Yancy, C. W. et al. $2013 \mathrm{ACCF} / \mathrm{AHA}$ guideline for the management of heart failure: a report of the American College of Cardiology Foundation/American Heart Association Task Force on Practice Guidelines. J. Am. Coll. Cardiol. 62, e147-239 (2013).

4. JCS Joint Working Group. Guidelines for rehabilitation in patients with cardiovascular disease (JCS 2012). Circ. J. 78, 2022-2093 (2014).

5. Bozkurt, B. et al. Cardiac rehabilitation for patients with heart failure: JACC expert panel. J. Am. Coll. Cardiol. 77, 1454-1469 (2021).

6. O'Connor, G. T. et al. An overview of randomized trials of rehabilitation with exercise after myocardial infarction. Circulation $\mathbf{8 0}$, 234-244 (1989).

7. Froelicher, V. et al. A randomized trial of exercise training in patients with coronary heart disease. JAMA 252, 1291-1297 (1984).

8. Schuler, G. et al. Myocardial perfusion and regression of coronary artery disease in patients on a regimen of intensive physical exercise and low fat diet. J. Am. Coll. Cardiol. 19, 34-42 (1992).

9. Kitzman, D. W. et al. Physical rehabilitation for older patients hospitalized for heart failure. N Engl. J. Med. 385, 203-216 (2021).

10. Agostoni, P. et al. Metabolic exercise test data combined with cardiac and kidney indexes, the MECKI score: A multiparametric approach to heart failure prognosis. Int. J. Cardiol. 167, 2710-2718 (2013).

11. Piepoli, M. F., Davos, C., Francis, D. P. \& Coats, A. J. ExTraMATCH collaborative exercise training meta-analysis of trials in patients with chronic heart failure (ExTraMATCH). BMJ 328, 189 (2004).

12. Carbone, S. et al. Peak oxygen consumption achieved at the end of cardiac rehabilitation predicts long-term survival in patients with coronary heart disease. Eur. Heart. J. Qual. Care Clin. Outcomes. https://doi.org/10.1093/ehjqcco/qcab032 (2021).

13. O'Connor, C. M. et al. Efficacy and safety of exercise training in patients with chronic heart failure: HF-ACTION randomized controlled trial. JAMA 301, 1439-1450 (2019).

14. Santhanakrishnan, R. et al. Atrial fibrillation begets heart failure and vice versa: Temporal associations and differences in preserved versus reduced ejection fraction. Circulation 133, 484-492 (2016).

15. Dries, D. L. et al. Atrial fibrillation is associated with an increased risk for mortality and heart failure progression in patients with asymptomatic and symptomatic left ventricular systolic dysfunction: A retrospective analysis of the SOLVD trials. Studies of left ventricular dysfunction. J. Am. Coll. Cardiol. 32, 695-703 (1998).

16. Wang, T. J. et al. Temporal relations of atrial fibrillation and congestive heart failure and their joint influence on mortality: The Framingham Heart Study. Circulation 107, 2920-2925 (2003).

17. Roy, D. et al. Rhythm control versus rate control for atrial fibrillation and heart failure. N Engl. J. Med. 358, 2667-2677 (2008).

18. Khan, M. N. et al. Pulmonary-vein isolation for atrial fibrillation in patients with heart failure. N Engl. J. Med. 359, 1778-1785 (2018).

19. Jones, D. G. et al. A randomized trial to assess catheter ablation versus rate control in the management of persistent atrial fibrillation in heart failure. J. Am. Coll. Cardiol. 61, 1894-1903 (2013).

20. Marrouche, N. F., Kheirkhahan, M. \& Brachmann, J. Catheter ablation for atrial fibrillation with heart failure. N Engl. J. Med. 379, 492 (2018).

21. Prabhu, S. et al. Regression of diffuse ventricular fibrosis following restoration of sinus rhythm with catheter ablation in patients with atrial fibrillation and systolic dysfunction: A substudy of the CAMERA MRI Trial. JACC Clin. Electrophysiol. 4, 999-1007 (2018).

22. Kirchhof, P. et al. Early rhythm-control therapy in patients with atrial fibrillation. N Engl. J. Med. 383, 1305-1316 (2020).

23. Reich, B. et al. Efficacy of extended, comprehensive outpatient cardiac rehabilitation on cardiovascular risk factors: A nationwide registry. Eur. J. Prev. Cardiol. 27, 1026-1033 (2020).

\section{Author contributions}

A.Y., and T.S. designed the study, conducted data analysis, and wrote the manuscript; T.I., M.A., K.Y., and Y.I. participated in data analysis; M.M., S.S, and K.A. participated in data collection. All authors have reviewed and approved the final version of the manuscript.

\section{Competing interests}

The authors declare no competing interests.

\section{Additional information}

Correspondence and requests for materials should be addressed to A.Y.

Reprints and permissions information is available at www.nature.com/reprints.

Publisher's note Springer Nature remains neutral with regard to jurisdictional claims in published maps and institutional affiliations. 
(c) (i) Open Access This article is licensed under a Creative Commons Attribution 4.0 International cc) License, which permits use, sharing, adaptation, distribution and reproduction in any medium or format, as long as you give appropriate credit to the original author(s) and the source, provide a link to the Creative Commons licence, and indicate if changes were made. The images or other third party material in this article are included in the article's Creative Commons licence, unless indicated otherwise in a credit line to the material. If material is not included in the article's Creative Commons licence and your intended use is not permitted by statutory regulation or exceeds the permitted use, you will need to obtain permission directly from the copyright holder. To view a copy of this licence, visit http://creativecommons.org/licenses/by/4.0/.

(C) The Author(s) 2021 